\title{
Estimator and Tests for Coefficient of Variation in Uniform Distribution
}

\section{Jamal Hoseini ${ }^{1 *}$ and Anwar Mohammadi ${ }^{2}$}

${ }^{1}$ Clinical Research Developmental Center, Kermanshah University of Medical Sciences, Kermanshah, Iran

${ }^{2}$ Mathematical Sciences Department, Tarbiat Modares University, Tehran, Iran

\begin{abstract}
Coefficient of variation has been widely used as a measure of precision of measurement equipment in particular for medical laboratories. The objective of this paper is to Inference for the coefficient of variation in uniform distributions. By using the two approaches of central limit theorem and generalized variable, confidence interval for coefficient of variation are considered. The coverage properties of the proposed confidence intervals for proposed two methods are assessment by simulation. An example using data of the approved metrological company from Iran Standard and Industrial Researches Organization is provided.
\end{abstract}

Keywords: Coefficient of variation; Uniform distribution; Generalized variable; Central limit theorem

\section{Introduction}

Evaluation and monitoring the precision of medical laboratory equipment are very important. Ignoring the monitoring and control of measurement errors in laboratory equipment may reduce the accuracy of experiment results. In addition, before calibrating the equipment, we must check the precision of measurements [1]. Measurement errors are divided in two parts: systematic and random. Precision depends only on the distribution of random errors [2]. According to ISO/IEC GUIDE 99, precision is the closeness of agreement between indications or measured quantity values obtained by replicate measurements on the same or similar objects under specified conditions and usually expressed numerically by measures of imprecision, such as standard deviation or coefficient of variation (CV) under the specified conditions of measurement [3]. Then, coefficient of variation has been widely used as a measure of precision of measurement equipment in particular for medical laboratories [2-4].

The coefficient of variation of a population is defined as the ratio of population standard deviation to the population mean, and then the coefficient of variation of $\mathrm{X}$ is given by:

$$
k=\frac{\sigma}{\mu}
$$

In order to draw inferences concerning the population $\mathrm{CV}$, it is necessary to make assumptions on the shape of the distribution of the population and to have a prior knowledge of the distribution of the estimator of population CV. An exact method for inferences about the coefficient of variation from a normal population based on the noncentral t distribution is available, but it is computationally cumbersome [5]. The first published approximation method for the construction of a CI for normal coefficient of variation was by McKay and David [6,7]. Vangel [8] provided a modification to McKay's method which was based on analysis of the distribution of a class of approximate pivotal quantities. Koopmans et al. [9] inferred the confidence intervals of the CV for normal and lognormal distributions. Sharma and Krishna [10] developed the asymptotic distribution of the reciprocal of $\mathrm{CV}$, which is called ICV, without making any assumptions about the distribution. Wong and $\mathrm{Wu}$ [11] developed small sample asymptotic method to obtain approximate confidence intervals for the coefficient of variation for both normal and no normal distributions. Pang et al. [12] proposed a simulation based Bayesian approach for constructing the interval estimation of the CV for Weibull, lognormal and gamma distributions
[12]. Forkman and Verrill [13] shown that McKay's approximation is type II noncentral beta distributed. Liang [14] studied the empirical Bayes estimation for the coefficient of variation in shifted exponential distributions. Mahmoudvand and Hassani [15] introduce two new estimators for the population coefficient of variation, in a normal distribution. Banik and Kibria [16] attempt has been made to estimating the population coefficient of variation by confidence intervals. But, the statistical tests in the literature not reported inferences about the coefficient of variation in uniform distribution. Therefore, it is of practical and theoretical importance to develop procedures for interval estimation and hypothesis testing for the coefficient of variation based on independent uniform samples.

In this paper, we introduce an unbiased estimator for the population coefficient of variation, in a uniform distribution.

\section{Estimators and Confidence Intervals for $\mathrm{CV}$}

Let $\mathrm{X}$ be a random variable having a uniform distribution with a probability density

$$
p(x \mid \mu, \sigma)=(2 \sqrt{3} \sigma)^{-1} I(\mu-\sqrt{3} \sigma<x<\mu+\sqrt{3} \sigma)
$$

where $-\infty<\mu<\infty, \sigma>0$, and $I(A)$ is the indicator function of the event A. Given $(\mu, \sigma), E_{(\mu, \sigma)}[X]=\mu, \operatorname{Var}_{(\mu, \sigma)}[X]=\sigma^{2}$. Thus, the CV of a uniform distribution is $\gamma \equiv \gamma(\mu, \sigma)=\sigma / \mu$. Our goal is to estimate $\gamma$.

Let $X_{l}, \ldots, X_{n}$ be a sample of size $\mathrm{n}(\geq 2)$ taken from a uniform distribution.

Let $X_{(l)}=\min \left(X_{1}, \cdots, X_{n}\right)$ and $X_{(n)}=\max \left(X_{1}, \ldots, X_{n}\right)$. Then $\left(Y_{1}, Y_{n}\right)$ is complete and sufficient for $(\mu, \sigma)$. Given $(\mu, \sigma)$,

$$
\hat{\mu}=\left(X_{(l)}+X_{(l)}\right) / 2 \text { and } \hat{\sigma}=\left(X_{(n)}+X_{(l)}\right) / 2 \sqrt{3}
$$

${ }^{*}$ Corresponding author: J. Hoseini, Clinical Research Developmental Center Kermanshah University of Medical Sciences, Kermanshah, Iran, Tel: +98 831 4276299; Fax: +98 831 4276299; E-mail: jhoseini2003@yahoo.com

Received May 16, 2012; Accepted June 25, 2012; Published June 25, 2012

Citation: Hoseini J, Mohammadi A (2012) Estimator and Tests for Coefficient of Variation in Uniform Distribution. J Biomet Biostat 3:149. doi:10.4172/21556180.1000149

Copyright: $\odot 2012$ Hoseini J, et al. This is an open-access article distributed under the terms of the Creative Commons Attribution License, which permits unrestricted use, distribution, and reproduction in any medium, provided the original author and source are credited. 
are the MLEs of $\mu$ and $\sigma$, respectively. Note that $E(\mu, \sigma)[\hat{\mu}]=\mu$, thus, $\hat{\mu}$ is unbiased estimator of $\mu$. But $\mathrm{E}_{(\mu, \sigma)}[\hat{\sigma}]=\frac{\mathrm{n}+1}{\mathrm{n}-1} \sigma$, then $\hat{\sigma}$ is biased estimator of $\sigma$. To generate a unbiased estimator from $\hat{\sigma}$, must use the statistic of $\frac{n+1}{n-1} \hat{\sigma}$. Also, $\hat{\mu}$ and $\frac{n+1}{n-1} \hat{\sigma}$ are the UMVUEs of $\mu$ and $\sigma$, respectively.

Let $T=\hat{\mu}=\left(X_{(l)}+X_{(n)}\right) / 2$ and $R=(2 \sqrt{3}) \hat{\sigma}=\left(X_{(n)}-X_{(l)}\right)$. Thus, given $(\mu, \sigma),(T, R)$, has a joint probability density

$$
\begin{aligned}
& p(t, r \mid \mu, \sigma)=\frac{n(n-1)}{(2 \sqrt{3} \sigma)^{n}} r^{\mathrm{n}-2} \\
& I(\mu-\sqrt{3} \sigma+r / 2<t<\mu+\sqrt{3} \sigma-r / 2) I(0<r<2 \sqrt{3} \sigma)
\end{aligned}
$$

Note that $\left(\frac{2 \sqrt{3}(n-2)}{n}\right)\left(\frac{T}{R}\right)$ is UMVUE of $1 / \gamma=\mu / \sigma[17]$.

Theorem 1: Let $X_{(1)}=\min \left(X_{1}, \ldots, X_{n}\right), X_{(n)}=\max \left(X_{1}, \ldots, X_{n}\right)$ and $\bar{X}=\sum_{i=1}^{n} x_{i} / n$. Then

$$
\frac{2 \sqrt{3} \bar{X}}{\left(X_{(n)}-X_{(1)}\right)}-\frac{\sqrt{n} \mu}{\sigma} \stackrel{d}{\rightarrow} N(0,1)
$$

Proof: Let $Z_{n}=\frac{\sqrt{n}(\bar{X}-\mu)}{\sigma}$, by central limit theorem, denote a standardized normal random variable. Then,

$$
\bar{X} \stackrel{d}{\rightarrow} N\left(\mu, \frac{\sigma^{2}}{n}\right)
$$

where,

$$
X_{(n)}-X_{(1)} \stackrel{p}{\rightarrow} 2 \sqrt{3} \sigma
$$

Thus,

$$
\begin{aligned}
& 2 \sqrt{3} \sigma\left(\frac{\bar{X}}{X_{(n)}-X_{(1)}}\right) \stackrel{d}{\rightarrow} N\left(\mu, \frac{\sigma^{2}}{n}\right) \\
& 2 \sqrt{3} \sigma\left(\frac{\bar{X}}{X_{(n)}-X_{(1)}}\right)-\mu \stackrel{d}{\rightarrow} N\left(0, \frac{\sigma^{2}}{n}\right) \\
& \frac{2 \sqrt{3} \sigma\left(\frac{\bar{X}}{X_{(n)}-X_{(1)}}\right)-\mu}{\sqrt{\frac{\sigma^{2}}{n}}} \stackrel{d}{\rightarrow} N(0,1)
\end{aligned}
$$

Hence

$$
2 \sqrt{3 n}\left(\frac{\bar{X}}{X_{(n)}-X_{(1)}}\right)-\frac{\sqrt{n} \mu}{\sigma} \stackrel{d}{\rightarrow} N(0,1) .
$$

Therefore, the $100(1-\alpha) \%$ confidence interval for $\tau$ based on Equation

$$
\frac{2 \sqrt{3} \overline{\mathrm{X}}}{X_{(n)}-X_{(1)}}-\frac{Z_{1-\frac{\alpha}{2}}}{\sqrt{n}} \leq \frac{\mu}{\sigma} \leq \frac{2 \sqrt{3} \overline{\mathrm{X}}}{X_{(n)}-X_{(1)}}+\frac{Z_{1-\frac{\alpha}{2}}}{\sqrt{n}}
$$

Theorem 2: Let $T=\hat{\mu}=\left(X_{(l)}+X_{(n)}\right) / 2$ and $R=(2 \sqrt{3}) \hat{\sigma}=\left(X_{(n)}-X_{(n)}\right)$ Then

$$
R_{\dot{e}}=\frac{t}{r} \frac{R}{\sigma}-\sqrt{3}\left(\frac{T-\mu+\sqrt{3} \sigma}{\sqrt{3} \sigma}\right)+\sqrt{3}
$$

Is generalized pivotal quantity for $\theta=1 / \psi=\mu / \sigma$. If $R_{\psi}=1 / R_{\theta}$, then $T_{\psi}=R_{\psi}-\psi$ is the generalized test variable for testing $H_{0}: \psi \geq \psi_{0}$ vs $H_{1}: \psi<\psi_{0}$,

$$
\begin{aligned}
& \text { Proof: } R_{\theta}=\frac{t}{r} \frac{R}{\sigma}-\sqrt{3}\left(\frac{T-\mu+\sqrt{3} \sigma}{\sqrt{3} \sigma}\right)+\sqrt{3} \\
& =\frac{t}{r}\left(2 \sqrt{3} \frac{X_{(n)}-X_{(1)}}{2 \sqrt{3} \sigma}\right)-\sqrt{3}\left(\frac{X_{(1)}-\mu+\sqrt{3} \sigma+X_{(n)}-\mu+\sqrt{3} \sigma}{2 \sqrt{3} \sigma}\right)+\sqrt{3} \\
& =\frac{t}{r}\left(2 \sqrt{3} U_{1}\right)-\sqrt{3}\left(U_{2}+U_{3}-1\right)
\end{aligned}
$$

where $U_{1} \sim \operatorname{Beta}(n-1,2)$, and $U_{2} \sim \operatorname{Beta}(1, n), U_{3} \sim \operatorname{Beta}(n, 1)$, then $R_{\theta}$ has a distribution free of $\mu$ and $\sigma$. Obviously, the observed value of $R_{\theta}$ as $T$ $=t$ and $R=\operatorname{ris} \theta$. Moreover, the distribution of $R_{\theta}$ does not depend on unknown parameters. Therefore, $R_{\theta}$ is a generalized pivot for $\theta$. The generalized pivot for $\psi=\sigma / \mu$ is $R_{\psi}=1 / R_{\theta}$. Note that $T_{\psi}$ satisfy the three conditions above: (1) For fixed t and $\mathrm{r}$, the distribution of $T_{\psi}$ is free of nuisance parameters; (2) $t_{\psi}$, the observed value of $T_{\psi}$ is 0 , and hence is free of any unknown parameters; and (3) $T_{\psi}$ is stochastically decreasing in $\psi$.

Therefore, $T_{\psi}$ is bonafide generalized pivotal variable for constructing confidence interval of and its quantiles may be used to construct confidence limits for $\psi=\sigma / \mu$.

For a given data set $x_{i}$ where $i=1,2, ., \mathrm{n}$, the generalized confidence intervals and the generalized P-values can be computed by the following steps:

1. Compute $t$ and $r$.

2. Generate $U_{1} \sim \operatorname{Beta}(n-1,2), \quad U_{2} \sim \operatorname{Beta}(1, n), \quad U_{3} \sim \operatorname{Beta}(n, 1)$, then compute $R_{\psi}$.

3. Repeat Step 2 a total $m$ times and obtain an array of R $\psi$ 's.

4. Rank this array of $R_{\psi}$ 's from small to large.

The 100ath percentile of $R_{\psi}$ 's, $R_{\psi}(\alpha)$ is an estimate of the lower bound of the one-sided

$100(1-\alpha)$ percent a confidence interval and $R_{\psi}(\alpha / 2) ; R_{\psi}(1-\alpha / 2)$ is a two-sided $100(1-\alpha)$ per cent confidence interval. The percentage that $R_{\psi}$ 's are less than or equal to $\psi_{0}$ is a Monte Carlo estimate of the generalized P-value for testing $\psi=\psi_{0}$ vs $\psi<\psi_{0}$.

\section{Comparison of the Methods}

Two approximation methods for the construction of CI for $\mathrm{k}$ were highlighted in Sec. 2. If a CI for CV is to be constructed, there is no clear guidance as to which of these methods has to be used. In particular, there is no information regarding relative performance of the CI from central limit theorem and generalized variable methods introduced in this article. In view of this, there is a need to compare these approximation methods in order to assess their performance and select one which is appropriate. The two approximate methods are compared in the construction of a CI for CV using simulation and real data sets.

\section{Simulation study}

Simulation studies are done to compare the length of the 95\% CI for $\mathrm{CV}$ obtained from central limit theorem and generalized variable methods. Data were generated from Uniform distribution with $\mathrm{CV}=$ $0.1,0.3,0.6,1$ and 3, and sample size $\mathrm{n}=10,25,50$ and 100. All simulations were performed using programs written in the $\mathrm{R}$ statistical software [13-15] with the number of simulation runs $M=10,000$ at level of significance $\alpha=0.05$ and 0.01 .Given the role that $\mathrm{m}$ (repeat frequent) in function of constructed confidence intervals by generalized 
variable method, Comparisons are done between confidence interval from central limit theorem method and confidence intervals from generalized variable methods with $\mathrm{m}=5,10,30,50,100$. The simulation results are shown in Tables 1-2, in which the following information, viz. The estimated coverage percentages of the confidence intervals from central limit theorem and generalized variable methods for a Uniform distribution at $\alpha=0.05$ and 0.01 , is presented respectively.

As can be seen the Tables 1 and 2 , all of the confidence intervals, for $95 \%$ confidence level only for two location that constructed confidence interval by central limit theorem method and eighteen location that constructed confidence interval by generalized variable method, have the most of coverage percentage. Also, for $99 \%$ confidence level only for five location that constructed confidence interval by central limit theorem method and five teen location that constructed confidence interval by generalized variable method, have the most of coverage percentage.

Effect of coefficient of variation value on confidence intervals: With a brief look at the results that can be made to the weakness of confidence interval by central limit theorem method for small values of the coefficient of variation. So that in both confidence level for the first three values of parameters of coefficient of variation (0.1, 0.3 and 0.6$)$, the highest of cover percentage to has been the confidence intervals constructed by generalized variable method with $m=100$. However, increasing the parameter of coefficient of variation, performance of confidence intervals of constructed to central limit theorem method is improved. But in general, performance of confidence intervals of constructed to central limit theorem method for small values of the coefficient of variation parameter is not suitable. But for larger values and confidence intervals of constructed to generalized variable method, its performance is improved.

Effect of sample size on confidence intervals: Note that the central limit theorem support of confidence intervals of constructed to central limit theorem method, Not unexpected that the confidence intervals in the high size of samples to show better performance. This issue carefully in both tables (1) and (2) can be clearly seen. So that for the five-coefficient of variations parameter value with increasing sample size clearly increase to coverage percentages of confidence interval of constructed to central limit theorem method. But it problem for confidence interval of constructed to generalized variable method is not seen. Then, does not appear to increase the sample size may have an effect on the performance improvement. Thus it appears that in practice the use of confidence interval of generalized variable method for a small sample size is much more reasonable.

Efficiency of repeat frequent on constructed confidence intervals by generalized variable method: With attention to results of simulation study the important role of this agent in the performance of confidence intervals is clearly visible. In all positions with increasing $\mathrm{m}$, coverage percentages of confidence interval of constructed to generalized variable method significantly increased.

\section{A real example}

The approved metrological company from Iran Standard and Industrial Researches Organization has conducted the study for the purpose of evaluation of precision for medical laboratory micropipettes according to NCCLS EP5-A2 and ISO 8655-6. CV is commonly presented in the report of the measurements. Samples were collect for three kinds of micropipettes which are common in medical diagnosis labs are used and are shown by A, B and C. A lab unit technician sampled the distilled water in a standard lab condition at the beginning of the work time and repeated the sampling two hours later. Overall, there were 40 runs in 28 consecutive days, and in every measurement 10 times sampling was conducted for each three kinds of micropipettes. The summary statistics of data collected from the three kinds of micropipettes of A, B and Care for every run given in Table 3. Initial review of 10 times of sampling in every run for any micropipette by Kolmogorov-Smirnov test shows that data follow a uniform distribution (Table 3). Therefore, we are interested in making inference about the coefficient of variation of every run based data of three kinds of micropipettes. Take 0.006 as a hypothetical value for the criteria of good precision for the measurements of micropipette according to ISO 8655-6. Upper limit of 95\% confidence interval for 40

\begin{tabular}{|c|c|c|c|c|c|c|c|}
\hline \multirow{3}{*}{$\mathrm{CV}\left(\frac{\sigma}{\mu}\right)$} & \multirow{3}{*}{ Sample Size (n) } & \multicolumn{6}{|c|}{ Coverage Percentage } \\
\hline & & \multirow{2}{*}{ Method $(I)$} & \multicolumn{5}{|c|}{ Method (II) } \\
\hline & & & $m=5$ & $m=10$ & $m=30$ & $m=50$ & $m=100$ \\
\hline \multirow{4}{*}{0.1} & 10 & 0.1267 & 0.6512 & 0.7906 & 0.9030 & 0.9205 & 0.9427 \\
\hline & 25 & 0.2493 & 0.6482 & 0.7962 & 0.9031 & 0.9247 & 0.9428 \\
\hline & 50 & 0.3820 & 0.6514 & 0.7939 & 0.9051 & 0.9212 & 0.9427 \\
\hline & 100 & 0.5309 & 0.6466 & 0.8005 & 0.9096 & 0.9294 & 0.9480 \\
\hline \multirow{4}{*}{0.3} & 10 & 0.4726 & 0.6664 & 0.8187 & 0.9296 & 0.9462 & 0.9650 \\
\hline & 25 & 0.6820 & 0.6638 & 0.8262 & 0.9299 & 0.9446 & 0.9575 \\
\hline & 50 & 0.7907 & 0.6699 & 0.8231 & 0.9330 & 0.9461 & 0.9590 \\
\hline & 100 & 0.8697 & 0.6761 & 0.8300 & 0.9329 & 0.9475 & 0.9611 \\
\hline \multirow{4}{*}{0.6} & 10 & 0.7256 & 0.6683 & 0.8242 & 0.9351 & 0.9445 & 0.9560 \\
\hline & 25 & 0.8427 & 0.6690 & 0.8270 & 0.9316 & 0.9431 & 0.9514 \\
\hline & 50 & 0.8977 & 0.6684 & 0.8323 & 0.9354 & 0.9434 & 0.9506 \\
\hline & 100 & 0.9257 & 0.6742 & 0.8360 & 0.9351 & 0.9436 & 0.9535 \\
\hline \multirow{4}{*}{1} & 10 & 0.8166 & 0.6468 & 0.7991 & 0.9132 & 0.9263 & 0.9447 \\
\hline & 25 & 0.8921 & 0.6538 & 0.8020 & 0.9146 & 0.9243 & 0.9388 \\
\hline & 50 & 0.9209 & 0.6576 & 0.8185 & 0.9175 & 0.9304 & 0.9434 \\
\hline & 100 & 0.9395 & 0.6630 & 0.8197 & 0.9200 & 0.9342 & 0.9461 \\
\hline \multirow{4}{*}{3} & 10 & 0.8636 & 0.7420 & 0.8594 & 0.9215 & 0.9263 & 0.9383 \\
\hline & 25 & 0.9206 & 0.6650 & 0.8083 & 0.9232 & 0.9328 & 0.9485 \\
\hline & 50 & 0.9332 & 0.6445 & 0.7941 & 0.9000 & 0.9122 & 0.9318 \\
\hline & 100 & 0.9458 & 0.6498 & 0.8033 & 0.9005 & 0.9143 & 0.9331 \\
\hline
\end{tabular}

Table 1: Coverage Percentage of $95 \%$ confidence Intervals from central limit theorem and generalized variable methods for a Uniform distribution. 
Citation: Hoseini J, Mohammadi A (2012) Estimator and Tests for Coefficient of Variation in Uniform Distribution. J Biomet Biostat 3:149. doi:10.4172/2155-6180.1000149

Page 4 of 5

\begin{tabular}{|c|c|c|c|c|c|c|c|}
\hline \multirow{3}{*}{$\operatorname{CV}\left(\frac{\sigma}{\mu}\right)$} & \multirow{3}{*}{ Sample Size (n) } & \multicolumn{6}{|c|}{ Coverage Percentage } \\
\hline & & \multirow{2}{*}{ Method $(I)$} & \multicolumn{5}{|c|}{ Method $(I I)$} \\
\hline & & & $m=5$ & $m=10$ & $m=30$ & $m=50$ & $m=100$ \\
\hline \multirow{4}{*}{0.1} & 10 & 0.1823 & 0.6693 & 0.8135 & 0.9357 & 0.9639 & 0.9816 \\
\hline & 25 & 0.3452 & 0.6649 & 0.8210 & 0.9348 & 0.9618 & 0.9802 \\
\hline & 50 & 0.5051 & 0.6711 & 0.8152 & 0.9403 & 0.9603 & 0.9794 \\
\hline & 100 & 0.6747 & 0.6695 & 0.8230 & 0.9418 & 0.9637 & 0.9818 \\
\hline \multirow{4}{*}{0.3} & 10 & 0.6013 & 0.6781 & 0.8442 & 0.9499 & 0.9728 & 0.9832 \\
\hline & 25 & 0.8002 & 0.6829 & 0.8462 & 0.9559 & 0.9706 & 0.9839 \\
\hline & 50 & 0.9001 & 0.6903 & 0.8439 & 0.9566 & 0.9732 & 0.9840 \\
\hline & 100 & 0.9484 & 0.6862 & 0.8413 & 0.9527 & 0.9703 & 0.9804 \\
\hline \multirow{4}{*}{0.6} & 10 & 0.8245 & 0.6756 & 0.8381 & 0.9506 & 0.9688 & 0.9784 \\
\hline & 25 & 0.9293 & 0.6892 & 0.8457 & 0.9547 & 0.9654 & 0.9760 \\
\hline & 50 & 0.9646 & 0.6836 & 0.8525 & 0.9524 & 0.9663 & 0.9762 \\
\hline & 100 & 0.9796 & 0.6856 & 0.8457 & 0.9476 & 0.9634 & 0.9733 \\
\hline \multirow{4}{*}{1} & 10 & 0.9009 & 0.6645 & 0.8188 & 0.9389 & 0.9571 & 0.9735 \\
\hline & 25 & 0.9583 & 0.6747 & 0.8328 & 0.9401 & 0.9579 & 0.9705 \\
\hline & 50 & 0.9789 & 0.6828 & 0.8419 & 0.9503 & 0.9626 & 0.9755 \\
\hline & 100 & 0.9864 & 0.6788 & .8338 & 0.9436 & 0.9604 & 0.9738 \\
\hline \multirow{4}{*}{3} & 10 & 0.9400 & 0.7501 & 0.8734 & 0.9391 & 0.9522 & 0.9681 \\
\hline & 25 & 0.9754 & 0.6814 & 0.8314 & 0.9507 & 0.9668 & 0.9800 \\
\hline & 50 & 0.9841 & 0.6608 & 0.8209 & 0.9297 & 0.9521 & 0.9722 \\
\hline & 100 & 0.9870 & 0.6668 & 0.8192 & 0.9287 & 0.9524 & 0.9722 \\
\hline
\end{tabular}

Table 2: Coverage Percentage of $99 \%$ confidence Intervals from central limit theorem and generalized variable methods for a Uniform distribution.

\begin{tabular}{|c|c|c|c|c|c|c|c|c|c|}
\hline \multirow{2}{*}{ RUN } & \multicolumn{3}{|c|}{ Micropipette A } & \multicolumn{3}{|c|}{ Micropipette B } & \multicolumn{3}{|c|}{ Micropipette C } \\
\hline & Minimum & Maximum & P-Value & Minimum & Maximum & P-Value & Minimum & Maximum & P-Value \\
\hline 1 & 0.04951 & 0.05001 & 0.508 & 0.04922 & 0.04978 & 0.200 & 0.04751 & 0.04800 & 0.533 \\
\hline 2 & 0.04960 & 0.05017 & 0.592 & 0.04932 & 0.05029 & 0.671 & 0.04706 & 0.04793 & 0.089 \\
\hline 3 & 0.04968 & 0.05018 & 0.198 & 0.04942 & 0.05006 & 0.422 & 0.04818 & 0.04858 & 0.436 \\
\hline 4 & 0.04983 & 0.05032 & 0.855 & 0.04938 & 0.05034 & 0.188 & 0.04813 & 0.04873 & 0.944 \\
\hline 5 & 0.04918 & 0.05025 & 0.017 & 0.04958 & 0.05008 & 0.042 & 0.04711 & 0.04794 & 0.472 \\
\hline 6 & 0.04930 & 0.05030 & 0.960 & 0.04963 & 0.05051 & 0.347 & 0.04740 & 0.04853 & 0.419 \\
\hline 7 & 0.04986 & 0.05018 & 0.919 & 0.04921 & 0.05056 & 0.227 & 0.04816 & 0.04979 & 0.965 \\
\hline 8 & 0.04933 & 0.05007 & 0.329 & 0.04951 & 0.05007 & 0.002 & 0.04909 & 0.04951 & 0.311 \\
\hline 9 & 0.04961 & 0.05012 & 0.886 & 0.04953 & 0.05023 & 0.230 & 0.04716 & 0.04860 & 0.288 \\
\hline 10 & 0.04955 & 0.05025 & 0.597 & 0.04910 & 0.04990 & 0.013 & 0.04808 & 0.04922 & 0.685 \\
\hline 11 & 0.04905 & 0.05004 & 0.060 & 0.04905 & 0.04985 & 0.496 & 0.04805 & 0.04938 & 0.213 \\
\hline 12 & 0.04983 & 0.05048 & 0.427 & 0.04910 & 0.05026 & 0.448 & 0.04821 & 0.04892 & 0.268 \\
\hline 13 & 0.04943 & 0.04988 & 0.115 & 0.04918 & 0.04975 & 0.893 & 0.04666 & 0.04765 & 0.920 \\
\hline 14 & 0.04961 & 0.05021 & 0.819 & 0.04920 & 0.05036 & 0.000 & 0.04794 & 0.04862 & 0.949 \\
\hline 15 & 0.04982 & 0.05017 & 0.065 & 0.04944 & 0.05070 & 0.690 & 0.04600 & 0.04774 & 0.155 \\
\hline 16 & 0.04978 & 0.05012 & 0.949 & 0.04933 & 0.05009 & 0.455 & 0.04726 & 0.04810 & 0.178 \\
\hline 17 & 0.04949 & 0.05003 & 0.897 & 0.04913 & 0.05003 & 0.097 & 0.04744 & 0.04881 & 0.012 \\
\hline 18 & 0.04985 & 0.05091 & 0.095 & 0.04891 & 0.05027 & 0.919 & 0.04999 & 0.05034 & 0.819 \\
\hline 19 & 0.04925 & 0.05018 & 0.267 & 0.04933 & 0.05016 & 0.710 & 0.04825 & 0.04924 & 0.396 \\
\hline 20 & 0.04918 & 0.04996 & 0.360 & 0.04914 & 0.05040 & 0.017 & 0.04810 & 0.04878 & 0.832 \\
\hline 21 & 0.04967 & 0.05033 & 0.130 & 0.04943 & 0.05014 & 0.998 & 0.04747 & 0.04863 & 0.329 \\
\hline 22 & 0.04975 & 0.05040 & 0.661 & 0.04918 & 0.05041 & 0.238 & 0.04844 & 0.04992 & 0.855 \\
\hline 23 & 0.04978 & 0.05055 & 0.044 & 0.04931 & 0.04972 & 0.917 & 0.04819 & 0.04923 & 0.530 \\
\hline 24 & 0.04935 & 0.05017 & 0.841 & 0.04934 & 0.05028 & 0.253 & 0.04869 & 0.04967 & 0.896 \\
\hline 25 & 0.04999 & 0.05063 & 0.709 & 0.04918 & 0.05013 & 0.010 & 0.04819 & 0.04991 & 0.433 \\
\hline 26 & 0.04925 & 0.05028 & 0.680 & 0.04936 & 0.05087 & 0.019 & 0.04819 & 0.04926 & 0.056 \\
\hline 27 & 0.04950 & 0.05004 & 0.022 & 0.04924 & 0.05032 & 0.143 & 0.04884 & 0.04957 & 0.500 \\
\hline 28 & 0.04947 & 0.05003 & 0.958 & 0.04924 & 0.04994 & 0.748 & 0.04649 & 0.04920 & 0.582 \\
\hline 29 & 0.04941 & 0.05025 & 0.999 & 0.04934 & 0.05136 & 0.000 & 0.04779 & 0.04850 & 0.431 \\
\hline 30 & 0.04988 & 0.05058 & 0.597 & 0.04911 & 0.05023 & 0.396 & 0.04730 & 0.04834 & 0.314 \\
\hline 31 & 0.04947 & 0.05010 & 0.673 & 0.04928 & 0.04973 & 0.707 & 0.04839 & 0.04908 & 0.243 \\
\hline 32 & 0.04971 & 0.05019 & 0.736 & 0.04911 & 0.04979 & 0.341 & 0.04715 & 0.04864 & 0.173 \\
\hline 33 & 0.04982 & 0.05025 & 0.022 & 0.04935 & 0.04990 & 0.536 & 0.04714 & 0.04802 & 0.975 \\
\hline 34 & 0.04970 & 0.05010 & 0.172 & 0.04914 & 0.05003 & 0.126 & 0.04731 & 0.04821 & 0.250 \\
\hline 35 & 0.04996 & 0.05033 & 0.041 & 0.04936 & 0.04984 & 0.193 & 0.04846 & 0.04934 & 0.786 \\
\hline 36 & 0.04954 & 0.05001 & 0.798 & 0.04940 & 0.04998 & 0.131 & 0.04868 & 0.04923 & 0.184 \\
\hline 37 & 0.04961 & 0.05011 & 0.413 & 0.04928 & 0.05025 & 0.020 & 0.04650 & 0.04854 & 0.001 \\
\hline 38 & 0.04963 & 0.04997 & 0.514 & 0.04935 & 0.04993 & 0.096 & 0.04902 & 0.04991 & 0.456 \\
\hline 39 & 0.04986 & 0.05056 & 0.673 & 0.04968 & 0.05009 & 0.044 & 0.04868 & 0.04938 & 0.748 \\
\hline 40 & 0.04989 & 0.05018 & 0.947 & 0.04959 & 0.05004 & 0.819 & 0.04786 & 0.04832 & 0.225 \\
\hline
\end{tabular}

Table 3: Summary statistics and P-value of Kolmogorov-Smirnov test for data collected from the three kinds of micropipettes of A, B and C are for every run. 
Citation: Hoseini J, Mohammadi A (2012) Estimator and Tests for Coefficient of Variation in Uniform Distribution. J Biomet Biostat 3:149. doi:10.4172/2155-6180.1000149

runs in three kinds of micropipettes by using two statistical methods are shown in Table 4.

\section{Conclusion}

In this paper, we propose two approaches for making inferences about the coefficient of variation in uniform distribution. These approaches utilize the concepts of generalized variables and central limit theorem. The performance of generalized variable method, alongside central limit theorem method, was assessed using simulation and real data. A comparison indicates that the coverage percentage of the proposed confidence interval of the generalized variable method is generally satisfactory, but it is computationally time consuming. Moreover, central limit theorem method takes a shorter time to calculate.

\section{Acknowledgements}

We are grateful to the Pishgaman Andazeshenasi Daghigh Company (approved metrological company from Iran Standard and Industrial Researches Organization), and especially to Dr. Hamid AlaviMajd, for providing us with precision of micropipettes the data.

\section{References}

1. Tholen DW, Kallner A, Kennedy JW, Krouwer JS, Meier K (2004) NCCLS EP5-A2 Evaluation of Precision Performance of Quantitative Measurement Methods; Approved Guideline, ( ${ }^{\text {nd }}$ edn), NCCLS, Pennsylvania, USA.

2. ISO 5725-1:1994. Accuracy (Trueness and Precision) of Measurement Methods and Results, Part 1: General Principles and Definitions. International Organization for Standardization, Geneva, Switzerland.

3. ISO/IEC GUIDE 99: (2007) International vocabulary of metrology- Basic and general concepts and associated terms (VIM). International Organization for Standardization, Switzerland.
4. ISO 2174-8:2004: Guidance for the Use of Repeatability, Reproducibility, and Trueness Estimates in Measurement Uncertainty Estimation. International Organization for Standardization, Geneva, Switzerland.

5. Lehmann EL, Romano JP (2005) Testing Statistical Hypotheses, ( $3^{\text {rd }}$ edn), New York, Springer.

6. McKay AT (1932) Distribution of the Coefficient of Variation and the Extended "t" Distribution. J R Stat Soc 95: 695-698.

7. David FN (1949) Note on the application of Fisher's k-statistics. Biometrika 36 : 383-393.

8. Vangel MG (1996) Confidence intervals for a normal coefficient of variation Am Stat 50: 21-26.

9. Koopmans LH, Owen DB, Rosenblatt JI (1964) Confidence intervals for the coefficient of variation for the normal and log normal distributions. Biometrika 51: 25-32.

10. Sharma KK, Krishna $H$ (1994) Asymptotic sampling distribution of inverse coefficient of variation and its applications. IEEE Trans Reliability 43: 630-633.

11. Wong ACM, Wu J (2002) Small sample asymptotic inference for the coefficient of variation: normal and nonnormal models. J Stat Plan Inference 104: 73-82.

12. Liu, Wei, Pang, Wan-Kai, Huang, Wei-Kuang (2006) Exact Confidence Bounds for the Coefficient of Variation of a Normally Distributed Population, International Journal of Statistics and Systems 1: 81-86.

13. Forkman J, Verrill S (2008) The distribution of McKay's approximation for the coefficient of variation, Statistics \& Probability Letters 78: 10-14.

14. Liang $T$ (2009) Empirical Bayes estimation of coefficient of variation in shifted exponential distributions. J Nonparametr Stat 21: 365-378.

15. Mahmoudvand R, Hassani $H$ (2009) Two new confidence intervals for the coefficient of variation in a normal distribution. J Appl Stat 36: 429-442.

16. Banik S, Golam Kibria BM (2011) Estimating the Population Coefficient of Variation by Confidence Intervals, Communications in Statistics - Simulation and Computation 40: 1236-1261.

17. Shao, Jun (2003) Mathematical Statistics ( $2^{\text {nd }}$ edn), New York, Springer. 